Bull. Austral. Math. Soc.

VoL. 37 (1988) [17-26]

\title{
SOME REPRESENTATION FORMULAE FOR ENTIRE FUNCTIONS OF EXPONENTIAL TYPE
}

\section{Clément Frappier}

We obtain some explicit formulae for series of the type

$$
\sum_{\substack{\nu=-\infty \\ \nu \neq 0}}^{\infty} \frac{f\left(\frac{2 \pi \nu}{\tau}\right)}{\nu^{r}}, \sum_{\substack{\nu=-\infty \\ \nu \neq 0}}^{\infty} \frac{(-1)^{\nu} f\left(\frac{\pi \nu}{\tau}\right)}{\nu^{r}}, r=2,3, \ldots
$$

where $f$ is an entire function of exponential type $\tau$, bounded on the real exis (and satisfying $h_{f}\left(\frac{\pi}{2}\right) \leqslant 0$ in the first case). These series are expressed in terms of the derivatives of $f$ and Bernoulli numbers. We examine the case where $f$ is a trigonometric polynomial which lead us, in particular, to a new representation of the associated Fejér mean.

\section{The general Case.}

In the book of Boas [2, Chapter 11] there are several inequalities involving entire functions of exponential type. Many of them are deduced from interpolation formulae. Let $f \in \mathcal{B}_{\tau}$, the class of entire functions of exponential type $\tau$, bounded on the real axis. The so called "cardinal series" [6]

$$
f(z)=\sin \tau z \sum_{\nu=-\infty}^{\infty} \frac{(-1)^{\nu} f\left(\frac{\pi \nu}{\tau}\right)}{\tau z-\pi \nu}
$$

converges uniformly in any bounded set of the complex plane. If $f(x)=O(|x|)$ for $x \in \mathbf{R}$, and if the sequence $f\left(\frac{\pi \nu}{\tau}\right), \nu \in \mathbf{Z}$, is bounded then $f(z)$ has the representation

$$
f(z)=\frac{f^{\prime}(0)}{\tau} \sin \tau z+f(0) \frac{\sin \tau z}{\tau z}+\tau z \sin \tau z \sum_{\substack{\nu=-\infty \\ \nu \neq 0}}^{\infty} \frac{(-1)^{\nu} f\left(\frac{\pi \nu}{\tau}\right)}{\pi \nu(\tau z-\pi \nu)} .
$$

Dividing by $\sin \tau z$ and differentiating both members of the resulting formula we obtain [5]

$$
f^{\prime}(z)-\tau f(z) \cot \tau z+-\tau \sin \tau z \sum_{\nu=-\infty}^{\infty} \frac{(-1)^{\nu} f\left(\frac{\pi \nu}{\tau}\right)}{(\tau z-\pi \nu)^{2}}
$$

Received 24 February 1987

Copyright Clearance Centre, Inc. Serial-fee code: 0004-9729/88 \$A2.00+0.00. 
It is clear that formula (3) can be differentiated to get explicit formulae involving higher derivatives of $f(z)$. However these formulae become complicated. But, as we shall see, they take a simple form in the case $z=0$. In the first place we impose on $f$ the additional hypothesis $h_{f}\left(\frac{\pi}{2}\right) \leqslant 0$, where $h_{f}(\Theta):=\varlimsup_{\rho \rightarrow \infty} \frac{\ln \left|f\left(\rho e^{i \Theta}\right)\right|}{\rho}$ is the Praghmen-Lindelöf indicator function. With that hypothesis it will be sufficient to interpolate at the points $\frac{2 \pi \nu}{\tau}$ for $\nu \in Z$; this is shown by:

ThEOREM 1. Let $f \in \mathcal{B}_{\tau}$ be such that $h_{f}\left(\frac{\pi}{2}\right) \leqslant 0$. For all integers $r \geqslant 2$ we have:

$$
r !\left(\frac{\tau}{2 \pi}\right)^{r} \sum_{\substack{\nu=-\infty \\
\nu \neq 0}}^{\infty} \frac{f\left(\frac{2 \pi \nu}{\tau}\right)}{\nu^{r}}=-\sum_{k=0}^{r}\left(\begin{array}{l}
r \\
k
\end{array}\right) B_{k}(i \tau)^{k} f^{(r-k)}(0)
$$

Here $B_{k}$ is the $k^{\text {th }}$ Bernoulli number defined by the generating function $\frac{x}{e^{z}-1}=$ $\sum_{k=0}^{\infty} \frac{B_{k}}{k !} z^{k},|z|<2 \pi$.

REMARK: The condition $h_{f}\left(\frac{\pi}{2}\right) \leqslant 0$ is necessary for the validity of Theorem 1 . To see that we may consider the function $f(z)=e^{-i \varepsilon z}, 0<\varepsilon \leqslant \tau$; we have $h_{f}\left(\frac{\pi}{2}\right)=\varepsilon$. If formula (4) were true for that function then it is readily seen that we would have, for $0<x \leqslant 1$, the equalities

$$
\frac{2(-1)^{\frac{r-2}{2}} r !}{(2 \pi)^{r}} \sum_{\nu=1}^{\infty} \frac{\cos (2 \pi x \nu)}{\nu^{r}}=\sum_{k=0}^{r}\left(\begin{array}{l}
r \\
k
\end{array}\right) B_{k}(-1)^{k} x^{r-k}
$$

in the case $r \equiv 0(\bmod 2)$, and

$$
\frac{2(-1)^{\frac{r-1}{2}} r !}{(2 \pi)^{r}} \sum_{\nu=1}^{\infty} \frac{\sin (2 \pi x \nu)}{\nu^{r}}=\sum_{k=0}^{r}\left(\begin{array}{l}
r \\
k
\end{array}\right) B_{k}(-1)^{k} x^{r-k}
$$

in the case $r \equiv 1 \bmod 2$. If $r=2$ then (5) would give

$$
1+6 x+6 x^{2}=\frac{6}{\pi^{2}} \sum_{\nu=1}^{\infty} \frac{\cos (2 \pi x \nu)}{\nu^{2}} \leqslant 1
$$

which is impossible for $x>0$. Similarly, if $r=3$ then

$$
2 x^{3}+3 x^{2}+x=\frac{3}{\pi^{3}} \sum_{\nu=1}^{\infty}-\frac{\sin (2 \pi x \nu)}{\nu^{3}} \leqslant \frac{3}{\pi^{3}} \sum_{\nu=1}^{\infty} \frac{2 \pi x \nu}{\nu^{3}}=x
$$

which is also impossible for $x>0$. Now we observe that differentiation of formula (5) (or(6)) gives us formula (6) (or (5)) where $r$ is replaced by $r-1$. Since it has already 
been observed that formulae (5) and (6) are not true for $r=2$ and $r=3$ respectively, we conclude that they cannot be true in an interval for $r \geqslant 2$.

Proof of Theorem 1: If $f \in \mathcal{B}_{\tau}$ belongs. to $L^{2}$ on the real axis then it has a representation of the form

$$
f(z)=\int_{-\tau}^{\tau} e^{i z t} \phi(t) d t
$$

where $\phi \in L^{2}(-\tau, \tau)$. If $f$ satisfies the additional hypothesis $h_{f}\left(\frac{\pi}{2}\right) \leqslant 0$ then it is not difficult to verify that the proof of $[2$, p.105] can be adapted to obtain a representation of the form

$$
f(z)=\int_{0}^{\tau} e^{e i z t} \phi(t) d t
$$

where $\phi \in L^{2}(0, \tau)$. Indeed, the hypothesis $h_{f}\left(\frac{\pi}{2}\right) \leqslant 0$ and $|f(x)| \leqslant M$ for $x \in \mathbf{R}$, imply [2, Theorem 6.2.4] that $|f(x+i y)| \leqslant M$ for $-\infty<x<\infty$ and $0 \leqslant y<\infty$, and this inequality (instead of $|f(x+i y)| \leqslant M e^{r y}$ ) gives us the required representation.

Now, if $f$ has the form (8) then

$$
\sum_{\substack{\nu=-\infty \\ \nu \neq 0}}^{\infty} \frac{f\left(\frac{2 \pi \nu}{\tau}\right)}{\nu^{r}}=\sum_{\substack{\nu=-\infty \\ \nu \neq 0}}^{\infty} \frac{1}{\nu^{r}} \int_{0}^{\tau} e^{2 \pi i \nu t / \tau} \phi(t) d t=\int_{0}^{\tau} \sum_{\substack{\nu=-\infty \\ \nu \neq 0}}^{\infty} \frac{e^{2 \pi i \nu t / \tau}}{\nu r} \phi(t) d t
$$

(by the Lebesgue dominated convergence theorem). If $B_{n}(x)$ is the $n t h$ Bernoulli polynomial, $B_{n}(x):=\sum_{k=0}^{n}\left(\begin{array}{l}n \\ k\end{array}\right) B_{k} x^{n-k}$, it is well-known $[1$, p.267] that

$$
B_{r}(x)=-\frac{r !}{(2 \pi i)^{r}} \sum_{\substack{\nu=-\infty \\ \nu \neq 0}}^{\infty} \frac{e^{2 \pi i \nu x}}{\nu^{r}}, 0 \leqslant x \leqslant 1,
$$

whence

$$
\sum_{\substack{\nu=-\infty \\ \nu \neq 0}}^{\infty} \frac{f\left(\frac{2 \pi \nu}{\tau}\right)}{\nu^{r}}=-\int_{0}^{\tau} \frac{(2 \pi i)^{r}}{r !} B_{r}\left(\frac{t}{\tau}\right) \phi(t) d t
$$

that is

$$
\frac{r !}{(2 \pi i)^{r}} \sum_{\substack{\nu=-\infty \\
\nu \neq 0}}^{\infty} \frac{f\left(\frac{2 \pi \nu}{r}\right)}{\nu^{r}}=-\int_{0}^{\tau} \sum_{k=0}^{r}\left(\begin{array}{l}
r \\
k
\end{array}\right) B_{k}\left(\frac{t}{\tau}\right)^{r-k} \phi(t) d t
$$

or, in view of $(8)$,

$$
\frac{r !}{(2 \pi i)^{r}} \sum_{\substack{\nu=-\infty \\
\nu \neq 0}}^{\infty} \frac{f\left(\frac{2 \pi \nu}{r}\right)}{\nu^{r}}=-\sum_{k=0}^{r}\left(\begin{array}{l}
r \\
k
\end{array}\right) B_{k} \frac{f^{(r-k)}(0)}{(i \tau)^{r-k}}
$$


This is the required formula whenever $f \in L^{2}(-\infty, \infty)$. To extend it to functions $f$ which are merely in $\mathcal{B}_{\tau}$ with $h_{f}\left(\frac{\pi}{2}\right) \leqslant 0$ we consider the functions $g_{\delta}(z):=$ $e^{i \delta z} \frac{\sin (\delta z)}{\delta z} f(z), \delta>0$. We have $g_{\delta} \in \mathcal{B}_{\tau+2 \delta}, h_{g \delta}\left(\frac{\pi}{2}\right)=h_{f}\left(\frac{\pi}{2}\right) \leqslant 0$ and $g_{\delta}$ belongs to $L^{2}(-\infty, \infty)$. Thus, using (10),

$$
r !\left(\frac{\tau+2 \delta}{2 \pi}\right)^{r} \sum_{\substack{\nu=-\infty \\
\nu \neq 0}}^{\infty} \frac{g_{\delta}\left(\frac{2 \pi \nu}{\tau+2 \delta}\right)}{\nu^{r}}=-\sum_{k=0}^{r}\left(\begin{array}{l}
r \\
k
\end{array}\right) B_{k}(i(\tau+2 \delta))^{k} g_{\delta}^{(r-k)}(0)
$$

and the result follows if we let $\delta \rightarrow 0$ (since $\left|g_{\delta}(x)\right| \leqslant \max _{-\infty<t<\infty}|f(t)|$ for $x \in \mathrm{R}$, the passage to the limit is easily justified by the Lebesgue dominated convergence theorem.) This completes the proof of Theorem 1 .

Let us apply the result of Theorem 1 to the function $g \in \mathcal{B}_{2 \tau}, g(z):=e^{i \tau z} f(z), f \in$ $\mathcal{B}_{\tau}$, which satisfies $h_{g}\left(\frac{\pi}{2}\right)=h_{f}\left(\frac{\pi}{2}\right)-\tau \leqslant 0$. If we use Leibniz's formula, substitute in (4), interchange the order of summation and use the formula $B_{m}\left(\frac{1}{2}\right)=\left(2^{-m+1}-1\right) B_{m}$ for $m=0,1,2, \ldots$ (obtainable from (9)), then we obtain

TheOREM 1'. Let $f \in \mathcal{B}_{\tau}$. For all integers $r \geqslant 2$ we have

$$
r !\left(\frac{\tau}{\pi}\right)^{r} \sum_{\substack{\nu=-\infty \\
\nu \neq 0}}^{\infty} \frac{(-1)^{\nu-1}}{\nu^{r}} f\left(\frac{\pi \nu}{\tau}\right)=\sum_{k=0}^{r}\left(\begin{array}{l}
r \\
k
\end{array}\right)\left(2-2^{k}\right)(1 \tau)^{k} B_{k} f^{(r-k)}(0) .
$$

As particular cases of $(11)$ we mention $(r=2)$

$$
\frac{6 \tau^{2}}{\pi^{2}} \sum_{\substack{\nu=-\infty \\ \nu \neq 0}}^{\infty} \frac{(-1)^{\nu-1}}{\nu^{2}} f\left(\frac{\pi \nu}{\tau}\right)=\tau^{2} f(0)+3 f^{\prime \prime}(0)
$$

a formula which may be obtained from (3) by evaluating

$$
\lim _{z \rightarrow 0}\left(\frac{f^{\prime}(z)-\tau f(z) \cot \tau z}{\sin \tau z}+\frac{f(0)}{\tau z^{2}}\right)
$$

and $(r=3)$

$$
\frac{6 \tau^{3}}{\pi^{3}} \sum_{\substack{\nu=-\infty \\ \nu \neq 0}}^{\infty} \frac{(-1)^{\nu-1}}{\nu^{3}} f\left(\frac{\pi \nu}{\tau}\right)=\tau^{2} f^{\prime}(0)+f^{\prime \prime \prime}(0)
$$

It is interesting to observe that the coefficient of the term $f^{(r-1)}(0)$ is always equal to zero in (11). 


\section{THE TRIGONOMETRIC POLYNOMIAL CASE}

A trigonometric polynomial $t$,

$$
t(z)=\sum_{j=-n}^{n} c_{j} e^{i j z}
$$

is an entire function of exponential type $n$, bounded on the real axis. It does not satisfy, in general, the condition $h_{t}\left(\frac{\pi}{2}\right) \leqslant 0$ but, as we shall see now, there is an interpolation formula which is closely related to (4) and (11) whenever $r=2$.

ThEOREM 2. Let $t(\Theta):=\sum_{j=-n}^{n} c_{j} e^{i j \Theta}$ be a trigonometric polynomial of degree $\leqslant n$ and $\sigma_{n}(t ; \Theta):=\sum_{j=-n}^{n}\left(1-\frac{|j|}{n}\right) c_{j} e^{i j \Theta}$ be the associated Fejér mean. We have

$$
\sigma_{n}(t ; \Theta) \equiv \frac{1}{n^{2}} t^{\prime \prime}(\Theta) \frac{1}{6}\left(5+\frac{1}{n^{2}}\right) t(\Theta)+\frac{1}{2 n^{2}} \sum_{k=1}^{n-1} \frac{t\left(\frac{2 \pi k}{n}+\Theta\right)}{\sin ^{2}\left(\frac{\pi k}{n}\right)}, n \geqslant 2
$$

The usual representation

$$
\sigma n(t ; \Theta)=\frac{1}{2 \pi} \int_{-\pi}^{\pi} t(\Theta+x)\left(\frac{\sin \left(\left(\frac{n+1}{2}\right) x\right)}{\sin \left(\frac{x}{2}\right)}\right)^{2} \frac{d x}{(n+1)}
$$

gives the inequality

$$
\left|\sigma_{n}(t ; \Theta)\right| \leqslant\|t\|_{(0,2 \pi)}, \quad \text { for } \Theta \in \mathbf{R}
$$

where

$$
\|t\|_{(0,2 \pi)}:=\max _{0<x<2 \pi}|t(x)| .
$$

As a particular case of (12) (for example $t(\Theta) \equiv 1$ ) we get

$$
\sum_{k=1}^{n-1} \frac{1}{\sin ^{2}\left(\frac{\pi k}{n}\right)}=\frac{n^{2}-1}{3}, \text { for } n \geqslant 2
$$

so that Theorem 2 has the immediate corollary:

COROLlaRY 1. For any trigonometric polynomial $t$, of degree $\leqslant n$, we have

$$
\left|\sigma_{n}(t ; \Theta)-\frac{1}{n^{2}} t^{\prime \prime}(\Theta)\right| \leqslant|t|_{(0,2 \pi)}, \Theta \in \mathbf{R}
$$

We note that the equality is possible in (14) for $t(\Theta)=a e^{-i n \Theta}+b+c e^{i n \Theta}$, where $a, b, c$, are any complex numbers. 
Proof of Theorem 2: . Let $s(z):=\sum_{j=-n}^{n} b_{j} e^{i j z}$ be a trigonometric polynomial of degree $\leqslant n$ such that $s(0)=s^{\prime}(0)=0$. The function $f(z):=s(z) / z^{2}$ is an entire function of exponential type $n$. Moreover,

$$
f(x)=O\left(\frac{1}{x^{2}}\right), \quad \text { as }|x| \rightarrow \infty .
$$

According to a known quadrature formula (see, for example, [4]) we have thus

$$
\int_{-\infty}^{\infty} f(x) d x=\frac{2 \pi}{n} \sum_{\nu=-\infty}^{\infty} f\left(\frac{2 \pi \nu}{n}\right)
$$

that is

$$
\int_{-\infty}^{\infty} \frac{s(x)}{x^{2}} d x=\frac{n}{2 \pi} \sum_{\substack{\nu=-\infty \\ \nu \neq 0}}^{\infty} \frac{s\left(\frac{2 \pi \nu}{n}\right)}{\nu^{2}}+\frac{\pi}{n} s^{\prime \prime}(0)
$$

Now,

$$
\begin{aligned}
\int_{-\infty}^{\infty} \frac{s(x)}{x^{2}} & =\int_{0}^{\infty} \frac{s(x)+s(-x)}{x^{2}} d x \\
& =\int_{0}^{\infty} \sum_{j=-n}^{n} b_{j} \frac{\left(e^{i j x}+e^{-i j x}\right)}{x^{2}} d x \\
& =\int_{0}^{\infty} \sum_{j=-n}^{n} b_{j} \frac{\left(e^{i j x / 2}-e^{-i j x / 2}\right)^{2}}{x^{2}} d x
\end{aligned}
$$

since $\sum_{j=-n}^{n} b_{j}=s(0)=0$, whence

$$
\begin{aligned}
\int_{-\infty}^{\infty} \frac{s(x)}{x^{2}} d x & =-4 \sum_{\substack{j=-n \\
j \neq 0}}^{n} b_{j} \int_{0}^{\infty}\left(\frac{\sin \left(\frac{j x}{2}\right)}{x}\right)^{2} d x \\
& =\pi \sum_{j=-n}^{-1} j b_{j}-\pi \sum_{j=1}^{n} j b_{j} \\
& =-\pi \sum_{j=-n}^{n}|j| b_{j} \\
& =-n \pi \sum_{j=-n}^{n}\left(1-\frac{|j|}{n}\right) b_{j} \text { since } s(0)=0 \\
& =-n \pi \sigma_{n}(s ; 0) .
\end{aligned}
$$


Thus, noting that $s\left(\frac{2 \pi \nu}{n}\right)=0$ if $\nu \equiv 0(\bmod n)$, we may write formula $(16)$ in the form

$$
\frac{1}{2 \pi} \sum_{\substack{\nu=-\infty \\ \nu \neq 0(n)}}^{\infty} \frac{s\left(\frac{2 \pi \nu}{n}\right)}{\nu^{2}}=\pi \sigma_{n}(s ; 0)-\frac{\pi}{n^{2}} s^{\prime \prime}(0) .
$$

If $t(z):=\sum_{j=-n}^{n} c_{j} e^{i j z}$ is an arbitrary trigonometric polynomial of degree $\leqslant n$ then the trigonometric polynomial $s(z)=t(z)+i\left(e^{i z}-1\right) t^{\prime}(0)-t(0)$ has a zero of multiplicity $\geqslant 2$ at $z=0$. Hence, using (17),

$$
\begin{aligned}
& \frac{1}{2 \pi} \sum_{\substack{\nu=-\infty \\
\nu \neq 0(n)}}^{\infty} \frac{t\left(\frac{2 \pi \nu}{n}\right)+i\left(e^{2 \pi i \nu / n}-1\right) t^{\prime}(0)-t(0)}{\nu^{2}} \\
& \quad=\pi \sigma_{n}(t ; 0)-\frac{\pi i}{n} t^{\prime}(0)-\pi t(0)-\frac{\pi}{n^{2}}\left(t^{\prime \prime}(0)-i t^{\prime}(0)\right) .
\end{aligned}
$$

Formula (4), with $r=2$, setting $f(z)=e^{i z}-1 \in \mathcal{B}_{n}$, gives

$$
\sum_{\substack{\nu=-\infty \\ \nu \neq 0}}^{\infty} \frac{e^{2 \pi i \nu / n}-1}{\nu^{2}}=2 \pi^{2}\left(\frac{1}{n^{2}}-\frac{1}{n}\right) ; \quad \text { also, } \sum_{\substack{\nu=-\infty \\ \nu \neq 0(n)}}^{\infty} \frac{1}{\nu^{2}}=\frac{\pi^{2}}{3}\left(1-\frac{1}{n^{2}}\right) .
$$

It then follows from (18) that

$$
\sigma_{n}(t ; 0)-t(0)+\frac{1}{6}\left(1-\frac{1}{n^{2}}\right) t(0)-\frac{1}{n^{2}} t^{\prime \prime}(0)=\frac{1}{2 \pi^{2}} \sum_{\substack{\nu-\infty \\ \nu \neq 0(n)}}^{\infty} \frac{t\left(\frac{2 \pi \nu}{n}\right)}{\nu^{2}}
$$

On the other hand,

$$
\sum_{\substack{\nu=-\infty \\ \nu \neq 0(n)}}^{\infty} \frac{t\left(\frac{2 \pi \nu}{n}\right)}{\nu^{2}}=\sum_{\mu=-\infty}^{\infty} \sum_{k=\mu n+1}^{(\mu+1) n-1} \frac{t\left(\frac{2 \pi k}{n}\right)}{k^{2}}=\sum_{\mu=-\infty}^{\infty} \sum_{k=1}^{n-1} \frac{t\left(\frac{2 \pi k}{n}\right)}{(k+\mu n)^{2}}
$$

The series being absolutely convergent we get

$$
\sum_{\substack{\nu=-\infty \\ \nu \neq 0(n)}}^{\infty} \frac{t\left(\frac{2 \pi \nu}{n}\right)}{\nu^{2}}=\sum_{k=1}^{n-1} \sum_{\mu=-\infty}^{\infty} \frac{1}{(k+\mu n)^{2}} \cdot t\left(\frac{2 \pi k}{n}\right)=\frac{\pi^{2}}{n^{2}} \sum_{k=1}^{n-1} \frac{t\left(\frac{2 \pi k}{n}\right)}{\sin ^{2}\left(\frac{\pi k}{n}\right)}
$$

where the last step uses the Mittag-Leffler expansion

$$
\sum_{\mu=-\infty}^{\infty} \frac{1}{(\mu+x)^{2}}=\left(\frac{\pi}{\sin \pi x}\right)^{2}
$$

with $x=k / n$. Formula (19) is thus equivalent to

$$
\sigma_{n}(t ; 0)-\frac{1}{6}\left(5+\frac{1}{n^{2}}\right) t(0)-\frac{1}{n^{2}} t^{\prime \prime}(0)=\frac{1}{2 n^{2}} \sum_{k=1}^{n-1} \frac{t\left(\frac{2 \pi k}{n}\right)}{\sin ^{2}\left(\frac{\pi k}{n}\right)}
$$

This completes the proof of Theorem 2 in the case $\Theta=0$. It is clear that (12) follows from (20) by translation. 


\section{The algebraic polynomial case.}

3.1. If $t(\Theta)=P\left(e^{i \Theta}\right)$, where $P(z):=\sum_{j=0}^{n} a_{j} z^{j}$ is an algebraic polynomial of degree $\leqslant n$, then $\sigma_{n}(t ; \Theta)=P\left(e^{i \Theta}\right)-\frac{1}{n} e^{i \Theta} P^{\prime}\left(e^{i \Theta}\right)$. Hence Theorem 2 admits the corollary:

COROLlary 2. For any algebraic polynomial $P$, of degree $\leqslant n$, we have

$$
z^{2} P^{\prime \prime}(z)-(n-1) z P^{\prime}(z)+\frac{\left(n^{2}-1\right)}{6} P(z)=\frac{1}{2} \sum_{k=1}^{n-1} \frac{P\left(z e^{2 \pi i k / n}\right)}{\sin ^{2}\left(\frac{\pi k}{n}\right)}, z \in C, n \geqslant 2 .
$$

If we write $z P^{\prime}(z)=\sum_{j=0}^{n} j a_{j} z^{j}$ and $z^{2} P^{\prime \prime}(z)=\sum_{j=0}^{n} j(j-1) a_{j} z^{j}$ and compare the coefficients in (21) then we see that Corollary 2 is equivalent to the equations $(n \geqslant 2)$ :

$$
\sum_{k=1}^{n-1} \frac{\cos \left(\frac{2 \pi k j}{n}\right)}{\sin \left(\frac{\pi k}{n}\right)}=2 j(j-n)+\frac{\left(n^{2}-1\right)}{3} \text { for } 0 \leqslant j \leqslant n
$$

and

$$
\sum_{k=1}^{n-1} \frac{\sin \left(\frac{2 \pi k j}{n}\right)}{\sin ^{2}\left(\frac{\pi k}{n}\right)}=0 \text { for } 0 \leqslant j \leqslant n \text {. }
$$

3.2. For any algebraic polynomial $P(z):=\sum_{j=0}^{n} a_{j} z^{j}$ the function $f(z)=P\left(e^{i z}\right)$ is in $\mathcal{B}_{n}$. Moreover, $h_{f}\left(\frac{\pi}{2}\right) \leqslant 0$. We have the formula (which may be proved easily by mathematical induction)

$$
f^{(k)}(z) \equiv i^{k} \sum_{j=1}^{k} S(k, j) e^{e j z} P^{(j)}\left(e^{i z}\right) \quad \text { for } k \geqslant 1
$$

where the $S(k, j)$ are Stirling numbers of the second kind, defined by the recurrence relation $S(1, k)=S(k, k)=1$ for $k \geqslant 1$ and $S(k, j)=j S(k, j-1)+S(k-1, j-1)$ for $1<j<k$. Thus formula (4) is applicable and noting that

$$
P\left(e^{i z}\right)=\sum_{k=0}^{n} \frac{P^{(k)}(1)}{k !}\left(e^{i z}-1\right)^{k}
$$

we obtain

$$
\begin{aligned}
\frac{r !}{(2 \pi i)^{r}} \sum_{\substack{\nu=-\infty \\
\nu \neq 0}}^{\infty} \sum_{k=0}^{n} \frac{P^{(k)}(1)\left(e^{2 \pi i \nu / n}-1\right)^{k}}{k ! \nu^{r}} & \\
& =-B_{r} P(1)-\sum_{k=1}^{r} \sum_{j=1}^{k} \frac{\left(\begin{array}{l}
r \\
k
\end{array}\right) B_{r-k}}{n^{k}} S(k, j) P^{(j)}(1)
\end{aligned}
$$

In both members of (26) we may interchange the order of summation; since the numbers $P^{(k)}(1)$ for $k=0,1,2, \ldots, n$ are arbitrary, we see that Theorem 1 admits, as a particular case, the following corollary: 
COROllary 3. Let

$$
b_{k, r, n}:=\frac{r !}{(2 \pi i)^{r}} \sum_{\substack{\nu=-\infty \\ \nu \neq 0}}^{\infty} \frac{\left(e^{\frac{2 \pi i \nu}{n}}-1\right)^{k}}{\nu^{r}}
$$

where $n \geqslant 1,0 \leqslant k \leqslant n$ and $r \geqslant 2$ are integers. We have $b_{o, r, n}=-B_{r}$,

$$
b_{k, r, n}=-k ! \sum_{j=k}^{r}\left(\begin{array}{l}
r \\
j
\end{array}\right) \frac{B_{r-j}}{n^{j}} S(j, k)
$$

for $1 \leqslant k \leqslant r$ and $b_{k, r, n}=0$ for $r<k$.

We may also apply formula (11) to the function $f(z)=P\left(e^{i z}\right)$. The same line of reasoning gives us the

COROLlaRY $3^{\prime}$. Let

$$
c_{k, r, n}:=\frac{r !}{(\pi i)^{r}} \sum_{\substack{\nu=-\infty \\ \nu \neq 0}}^{\infty} \frac{(-1)^{\nu-1}}{\nu^{r}}\left(e^{\frac{\pi i \nu}{n}}-1\right)^{k}
$$

where $n \geqslant 1,0 \leqslant k \leqslant n$ and $r \geqslant 2$ are integers. We have $c_{o, r, n}=\left(2-2^{r}\right) B_{r}$,

$$
c_{k, r, n}=k ! \sum_{j=k}^{r}\left(\begin{array}{l}
r \\
j
\end{array}\right) \frac{\left(2-2^{r-j}\right)}{n^{j}} B_{r-j} S(j, k),
$$

for $1 \leqslant k \leqslant r$ and $c_{k, r, n}=0$ for $r<k$.

It is to be noted that formula (27) is, for $r \leqslant k \leqslant n$, a consequence of the quadrature formula (15); we need only consider, in (15), the function $f(z)=\frac{\left(e^{i z}-1\right)^{k}}{z^{r}}$ which is an element of $\mathcal{B}_{n}$. However, in the case $k<r$, that function is not an entire function so that formula (15) is not applicable directly.

\section{OTHER OBSERVATIONS.}

Formula (4) could have been proved by other methods.

4.1. By mathematical induction. Suppose that formula (4) is proved for $r=2$. With the hypothesis that (4) is true for a given $r>2$ we may apply Theorem 1 to the function $F(z):=\frac{f(z)-f(0)}{z}$; it is a matter of simple computation to see then that $(4)$ is true with $r+1$ instead of $r$. To prove formula (4) with $r=2$ we may use the same line of proof as in the text or proceed as in 4.3 , below. 
4.2. With Taylor expansion. Here again we must suppose that formula (4) is established for $r=2$. We use the remainder term in the integral form to obtain, for $r \geqslant 3$,

$$
\sum_{k=0}^{r-3} \frac{f^{(k)}(0)}{k !}\left(\frac{2 \pi \nu}{\tau}\right)^{k}=f\left(\frac{2 \pi \nu}{\tau}\right)-\int_{0}^{\frac{2 \pi \nu}{\tau}} \frac{f^{(r-2)}(t)}{(r-3) !}\left(\frac{2 \pi \nu}{\tau}-t\right)^{r-3} d t .
$$

We substitute the righthand member of (29) in

$$
\sum_{k=0}^{r-3} \sum_{\substack{\nu=-\infty \\
\nu \neq 0}}^{\infty} \frac{f^{(k)}(0)}{k ! \nu^{r-k}}\left(\frac{2 \pi}{\tau}\right)^{k}=-\frac{1}{r !} \sum_{k=0}^{r-3}\left(\begin{array}{l}
r \\
k
\end{array}\right) B_{r-k}(2 \pi i)^{r-k} f^{(k)}(0)
$$

which leads us to (4) in the case $r \geqslant 3$.

4.3. By approximation. In the first place we must prove (4) when $f(z)=Q\left(e^{i z}\right)$, where $Q$ is a polynomial having a zero of multiplicity $\geqslant r$ at the point 1 . In general we approximate $f$ by polynomials of the kind considered in [4] and use (15) with an appropriate $n$. After some lengthy calculation we are led to a formula which turns out to be equivalent to (4). We omit the details of that proof but we observe that the coefficient of $f^{(r-1)}(0)$ appears in the form

$$
C \int_{-\infty}^{\infty} \frac{(\sin x)^{r-1} \sin (r-1) x}{x^{r}} d x
$$

for some computable constant $C$. Thus a conjunction of two proofs shows that

$$
\int_{-\infty}^{\infty} \frac{(\sin x)^{r-1} \sin (r-1) x}{x^{r}} d x=\pi, \text { for } r=2,3,4, \ldots
$$

\section{REFERENCES}

[1] T.M. Apostol,, Introduction to Analytic number theory (Springer-Verlag, New York, 1976).

[2] R.P. Boas, Entire Functions (Academic Press, New York, 1954).

[3] C. Frappier, 'Some inequalities for trigonometric polynomials', J. Austral. Math. Soc. Ser. A. 39 (1985), 216-226.

[4] C. Frappier and Q.I. Rahman, 'Une formule de quadrature pour les fonctions entières de type exponentiel', Ann. Sci. Math. Québec 10 (1986), 17-26.

[5] A.J. Macintyre, 'Laplace's transformation and integral functions', Proc. London Math. Soc (2) 45 (1938), 1-20.

[6] J.M. Whittaker, 'Interpolatory function theory', in Cambridge Tracts in Math. and Math. Physics 33 (Cambridge University Press, Cambridge, 1935).

Départment de Mathématiques Appliquées,

École Polytechnique de Montréal,

Campus de l'Université de Montréal,

Case Postale 6079, Succursale "A",

Monteéal, Québec, H3C 3 A7

Canada 